\title{
Spontaneous Adhesion of DOPA and Tryptophan Functionalized PEG to Polystyrene Nanobeads: An EPR Study Yaman GÖKSEL $L^{1, a}$, İklima KıRPAT ${ }^{1, b}$ and Yasar AKDOGAN ${ }^{1, c}$
}

\author{
${ }^{1}$ Izmir Institute of Technology, Materials Science and Engineering Department, İzmir, Turkey \\ ayamangoksel@iyte.edu.tr, biklimakirpat@gmail.com, cyasarakdogan@iyte.edu.tr
}

Keywords: Mussel adhesion, DOPA, EPR spectroscopy

\begin{abstract}
Wet adhesion is achieved by mussels so naturally. Their adhesion mechanism has inspired scientists to obtain wet adhesives for a long time. The amino acid 3,4dihydroxyphenylalanine (DOPA) produced by mussels adheres to different types of surfaces and also contributes to cohesive interactions. Here, we showed the spontaneous adhesion of DOPA functionalized four armed poly(ethylene glycol) (PEG) polymer to spin labeled polystyrene (SL-PS) nanosurfaces by electron paramagnetic resonance (EPR) spectroscopy. In addition to DOPA, adhesion property of another amino acid of tryptophan (Trp) was studied. Trp attached four armed PEG polymers did not adhere to the surface of SL-PS in the force free condition. However, two armed DOPA and two armed Trp functionalized PEG adhere to the PS.
\end{abstract}

\section{Introduction}

Marine mussels are able to attach to different surfaces in water in spite of the waves, winds, birds, etc. They can achieve strong and durable binding to a variety of surfaces using a bundle of threads and plaques which are composed of mostly mussel foot proteins (Mfps) [1]. Studies have shown that a dozen of Mfps are responsible for both adhesive and cohesive interactions [1, 2]. The common feature of these Mfps is containing an unusual amino acid of 3,4-dihydroxyphenylalanine (DOPA), formed through posttranslational modification of tyrosine amino acid [1]. The DOPA can bind to various surfaces via hydrogen bonding, metal-oxygen bonding and hydrophobic bonding, and also it plays an important role in intermolecular crosslinking [3]. DOPA is readily oxidized at alkaline $\mathrm{pH}$ values or in the presence of oxidizing agents for the curing of the adhesive [4].

Since DOPA has a special function for the adhesion mechanism of mussels, we have attached four DOPA molecules to the end of four armed poly(ethylene glycol) (PEG) polymer (PEG$\left.(\mathrm{DOPA})_{4}\right)$ [5]. In addition to DOPA, we have also studied the effect of another amino acid of tryptophan (Trp) on the adhesion mechanism [5]. Therefore, tryptophan functionalized four armed PEG polymer (PEG-(Trp) $)_{4}$ ) and a combination of DOPA and Trp functionalized (Trp) 2 -PEG$(\text { DOPA })_{2}$ were synthesized.

In the presence of water, strong hydration layers hinder the adhesion $[5,6]$. Especially in the force free condition, hydration repulsive forces must be overcome for the attachment. In order to determine the spontaneous wet adhesion, we have introduced electron paramagnetic resonance (EPR) spectroscopy in our previous studies [5,6]. EPR spectroscopy is a non-destructive technique to characterize the paramagnetic centers. Therefore, this technique is useful for studying metal complexes, inorganic and organic radicals, protein structures, drug delivery and surface defects, etc. [7-13]. Spin labeling of polystyrene (PS) in combination with EPR technique allows us to monitor the surface of nanobeads in solution $[5,6]$. The EPR line shape of a paramagnetic sample strictly depends on the dynamics behavior of the spin center. The rotational motion of spin labels on the surface of nanobeads is affected by the polymer adhesion. If the polymer covers the surface, this restricts the dynamics behavior of spin labels and changes the EPR line shape. Thus, EPR spectroscopy is able to determine the spontaneous adhesion in water. 


\section{Experimental}

The synthesis of PEG-(DOPA) $)_{4}$, PEG-(Trp) 4 and (Trp) $)_{2}$-PEG-(DOPA) $)_{2}$ were prepared as described in published procedures [5]. For DOPA attachment, the amine group of L-DOPA was protected with tertbutyloxycarbonyl (Boc) group and then the product of N-Boc-L-DOPA was reacted with PEG-( $\left(\mathrm{NH}_{2}\right)_{4}(10 \mathrm{kDa})$. In this study, the attached DOPA amino acids to PEG are all protected. ${ }^{1} \mathrm{H}$ NMR $\left(400 \mathrm{MHz}, \mathrm{CDCl}_{3}\right) \delta: 6.77(\mathrm{t}, 8 \mathrm{H}), 6.57(\mathrm{~d}, 4 \mathrm{H}), 6.15(\mathrm{~s}, 4 \mathrm{H}), 4.21(\mathrm{~s}, 4 \mathrm{H}), 3.81$ $(\mathrm{t}, 8 \mathrm{H}), 3.64-3.45(\mathrm{~m}, 896 \mathrm{H}), 3.00(\mathrm{~d}, 8 \mathrm{H}), 2.72(\mathrm{t}, 8 \mathrm{H}), 1.41(\mathrm{~s}, 36 \mathrm{H})$.

For PEG-(Trp $)_{4}$, PEG- $(\mathrm{OH})_{4}(10 \mathrm{kDa})$ and p-nitrophenyl chloroformate were mixed to obtain PEG-(p-nitrophenyl carbonate) $)_{4}$. Next, tryptophan and PEG-(p-nitrophenyl carbonate) $)_{4}$ were mixed to get PEG-(Trp) $)_{4}{ }^{1} \mathrm{H}$ NMR (400 MHz, DMSO-d6) $\delta: 10.75(\mathrm{~s}, 4 \mathrm{H}), 7.49$ (d, 4H), 7.30 (d, 4H), $7.11(\mathrm{~s}, 4 \mathrm{H}), 7.03(\mathrm{t}, 4 \mathrm{H}), 6.94(\mathrm{t}, 4 \mathrm{H}), 4.14-4.09(\mathrm{~m}, 8 \mathrm{H}), 3.48(\mathrm{~m}, 896 \mathrm{H}), 3.13(\mathrm{~d}, 8 \mathrm{H})$.

For (Trp) $)_{2}$-PEG-(DOPA $)_{2},\left(\mathrm{NH}_{2}\right)_{2}$-PEG-(OH) 2 (10 kDa) was transformed into $(\mathrm{H}-\mathrm{N}-\mathrm{Boc})_{2}$-PEG$(\mathrm{OH})_{2}$ using di-tert-butyl dicarbonate in the presence of triethyl amine. Then, with an ratio of 2:1:2 Trp:PEG:DOPA, first tryptophan and followed by DOPA amino acids were attached to the four armed PEG polymers just like the processes described above. ${ }^{1} \mathrm{H}$ NMR (400 MHz, DMSO-d6) $\delta$ : 7.49 (d, 2H), 7.29 (d, 2H), 7.11- 7.09 (m, 2H), 7.044-7.007 (m, 2H), 6.99-6.92 (m, 2H), 6.80-6.78 $(\mathrm{m}, 4 \mathrm{H}), 6.58-6.55(\mathrm{~m}, 232 \mathrm{H}), 6.54-6.43(\mathrm{~m}, 2 \mathrm{H}), 3.99-3.92(\mathrm{~m}, 10 \mathrm{H}), 3.48(896 \mathrm{H}), 3.20-3.17(\mathrm{~m}$, $8 \mathrm{H}), 2.99(\mathrm{t}, 4 \mathrm{H}), 1.29(\mathrm{~s}, 18 \mathrm{H})$.

Spin labeled polystyrene (SL-PS) nanobeads were prepared according to the previous studies [5, 6]. Amine modified polystyrene nanobeads $(60 \mathrm{~nm})$ were mixed with 4-carboxy-Tempo and 1ethyl-3-(3-dimethylaminopropyl) carbodiimide in 0.2 M MES buffer ( $\mathrm{pH} 3.0$ ) for one day at room temperature (RT). The resulting SL-PS was purified in centrifuge concentrator with washing several times with MES buffer. SL-PS was mixed with different concentrations of PEG- $\left(\mathrm{NH}_{2}\right)_{4}$, $(\operatorname{Trp})_{2}$ - $^{-}$ PEG-(DOPA) $)_{2}$ and PEG-(DOPA) $)_{4}$ with a volume ratio of 1:1 in MES buffer at $\mathrm{pH}$ 3.0. Acidic medium was used to avoid DOPA oxidation. X-band EPR measurements were done using a CMS 8400 (Adani) benchtop spectrometer at RT. All spectra were normalized to the intensities of the middle signals and also they were simulated using the Matlab-based Easyspin 4.5.5 software package [14]. UV-Vis spectra were obtained from UV-2550 UV-Visible spectrometer (Schimadzu, Japan).

\section{Results and Discussion}

Fig. 1 (A) shows UV-Vis spectra of functionalized four armed PEG polymers and the precursor sample of PEG-( $\left.\mathrm{NH}_{2}\right)_{4}$ in $0.2 \mathrm{M}$ MES buffer at $\mathrm{pH}$ 3.0. As expected, PEG-( $\left.\mathrm{NH}_{2}\right)_{4}$ did not show any absorption signals. However, after addition of DOPA molecules, a strong absorption signal at $278 \mathrm{~nm}$ was detected. Similarly, after addition of Trp amino acids, a broader absorption signal compared to DOPA signal at $279 \mathrm{~nm}$ was observed. These results show the attachment of DOPA and Trp to the four armed PEG polymers. In addition, both DOPA and Trp were attached to the same PEG polymer, and its UV-Vis spectrum shows both absorption features of DOPA and Trp. UV-Vis spectrum of (Trp) $)_{2}$-PEG-(DOPA) $)_{2}$ also shows the ratio of Trp and DOPA on the PEG polymer. Addition of $45 \%$ of UV-Vis spectrum of PEG-(DOPA) $)_{4}$ and $55 \%$ of UV-Vis spectrum of PEG-(Trp) ${ }_{4}$ gives the UV-Vis spectrum of (Trp) $)_{2}$-PEG-(DOPA) $)_{2}$ (Fig. 1 (B)). Threfore, four arms of each PEG polymer were successfully funtionalized with two Trp and two DOPA molecules. ${ }^{1} \mathrm{H}$ NMR results also proved the attachment of DOPA and Trp to PEG polymers. 
(A)

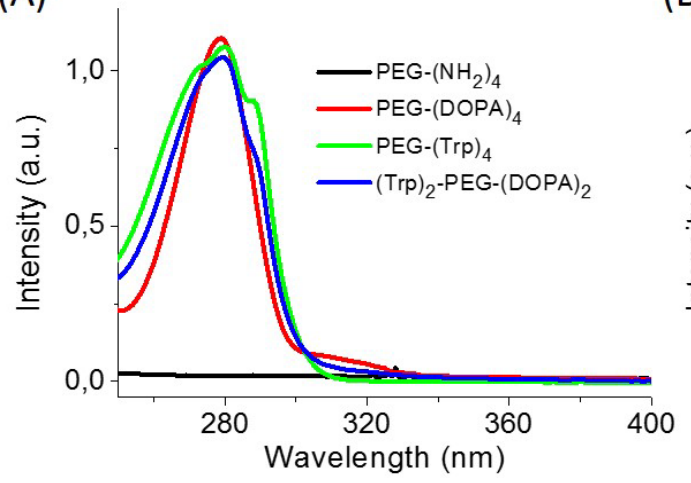

(B)

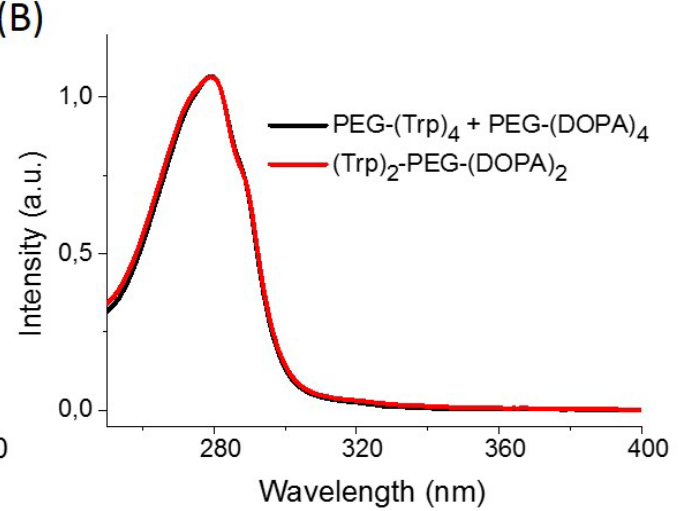

Fig. 1. (A) UV-Vis spectra of PEG-(NH $)_{4}$, PEG-(DOPA) $)_{4}$, PEG-(Trp) $)_{4}$ and (Trp) $)_{2}$-PEG-(DOPA) in $0.2 \mathrm{M}$ MES buffer at $\mathrm{pH} 3.0$. (B) Comparison of (Trp) $)_{2}-\mathrm{PEG}-(\mathrm{DOPA})_{2}$ and the sum of $45 \%$ of UV-Vis spectrum of PEG-(DOPA) ${ }_{4}$ and $55 \%$ of UV-vis spectrum of PEG-(Trp) $)_{4}$ from (A).

In addition to the adhesive function of DOPA molecule, DOPA is able to form inter molecular crosslinks. DOPA molecules can form a metal coordinated network in the presence of iron $\left(\mathrm{Fe}^{3+}\right)$ ions or can form covalent coordination between DOPA molecules in the presence of oxidizing agents [1]. Here, we showed the gel formation of (Trp) $)_{2}$-PEG-(DOPA) $)_{2}$ and PEG-(DOPA) polymers in the presence of bis(tetrabutylammonium) dichromate in $0.2 \mathrm{M} \mathrm{MES}$ buffer at pH 3.0. Dichromate $\left(\mathrm{Cr}_{2} \mathrm{O}_{7}{ }^{2-}\right)$ ions oxidize the DOPA molecules and form a covalently cross linked DOPA network. Fig. 2 shows the gel formation of (Trp) $)_{2}$-PEG-(DOPA) $)_{2}$ and PEG-(DOPA) $)_{4}$ in the presence of $\mathrm{Cr}_{2} \mathrm{O}_{7}{ }^{2-}$ ions. Since the number of DOPA is higher in PEG-(DOPA) $)_{4}$, the gel formation is completed fast (Fig. 2 (D)). For the (Trp) $)_{2}$-PEG-(DOPA) $)_{2}$, the gel formation is slower compared to the gel formation of PEG-(DOPA), and its gel also includes non gel part (Fig. 2 (C)). This also proves the success of synthesizing Trp and DOPA functionalized PEG polymers. As expected, PEG-(Trp) ${ }_{4}$ does not form gel with $\mathrm{Cr}_{2} \mathrm{O}_{7}{ }^{2-}$ ions (Fig. 2 (B)).

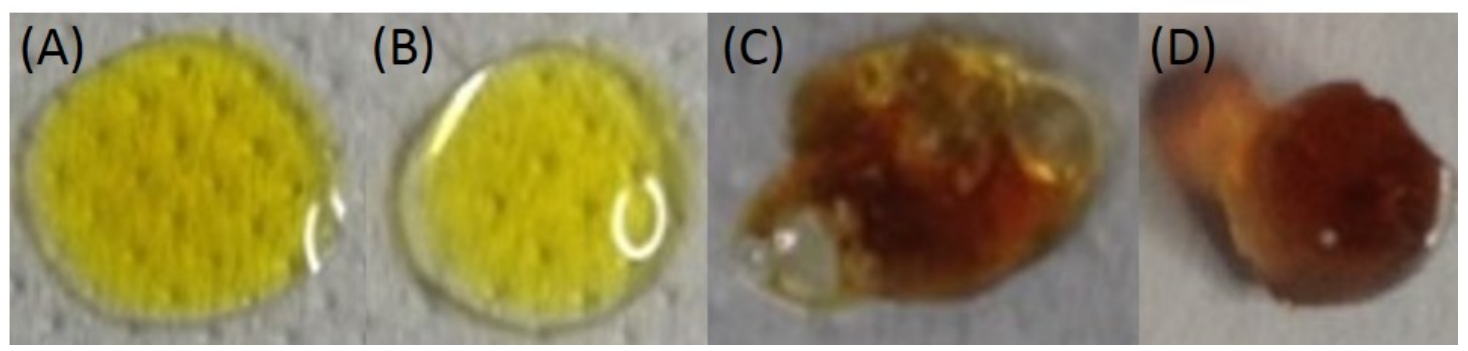

Fig. 2. Bis(tetrabutylammonium) dichromate in 0.2 M MES buffer at pH 3.0 (A), after addition of bis(tetrabutylammonium) dichromate to PEG-(Trp) ${ }_{4}$ solution (B), to (Trp) $)_{2}-\mathrm{PEG}-(\mathrm{DOPA})_{2}$ solution (C) and to PEG-(DOPA) 4 solution in 0.2 M MES buffer at $\mathrm{pH}$ 3.0.

In solution, dynamics behavior of paramagnetic molecules can be studied by EPR spectroscopy [5]. Since EPR lineshape is very sensitive to the rotational motion of the spin center, free spin labels (SL) and bound SL on PS nanoparticles must be different. The free SL shows three sharp EPR hyperfine lines because of the fast isotropic motion with a rotational correlation time 20 ps. However, the rotational motion of the bound SL is restricted therefore its EPR lineshape has broad hyperfine lines with a longer rotational correlation time 3 ns. Here, we characterized the adhesive properties of DOPA and Trp functionalized PEG polymers by using EPR spectroscopy in terms of dynamic motions of spin labels. Fig. 3 shows the EPR spectra of SL-PS before and after addition of PEG-(Trp) $)_{4},(\operatorname{Trp})_{2}$-PEG-(DOPA) $)_{2}$ and PEG-(DOPA) $)_{4}$. In Fig. 3 (A), the EPR spectrum of SL-PS does not change after addition of PEG-(Trp) $)_{4}$ with $22 \mathrm{mg} / \mathrm{mL}$ and $90 \mathrm{mg} / \mathrm{mL}$ concentrations. This means that PEG-(Trp) $)_{4}$ does not bind to surface of PS and spin labels on the PS are not affected from the PEG-(Trp) $)_{4}$ in the solution. Addition of both Trp and DOPA functionalized PEG polymers, (Trp) $)_{2}$-PEG-(DOPA) $)_{2}$, to the SL-PS suspension gradually changes the EPR spectrum of SL-PS (Fig. 3 (B)). A second type of spectrum nearby the spectrum of the bare SL-PS appears. 
Explicitly, a signal at $333.5 \mathrm{mT}$ shows the formation of this new type of spectrum. This new spectrum comes from the covered spin labels on the polystyrene. Therefore, its rotational motion is more restricted compared to the rotational motion of spin labels on the bare polystyrene. Simulations showed that rotational correlation time of covered SL is about 10 ns (5). Since PEG$(\operatorname{Trp})_{4}$ does not bind to SL-PS surface, DOPA plays a crucial role for the adhesion of (Trp) $)_{2}$-PEG(DOPA $)_{2}$ to the SL-PS surface. In order to show the adhesion role of DOPA, PEG-(DOPA) $)_{4}$ was added to SL-PS suspension. For both $22 \mathrm{mg} / \mathrm{mL}$ and $90 \mathrm{mg} / \mathrm{mL}$ of PEG-(DOPA) 4 concentrations, the second type of spectrum was observed clearly. There is a signal at $333.5 \mathrm{mT}$ appears very strongly which is originated from covered spin labels by PEG-(DOPA) 4 .

(A)

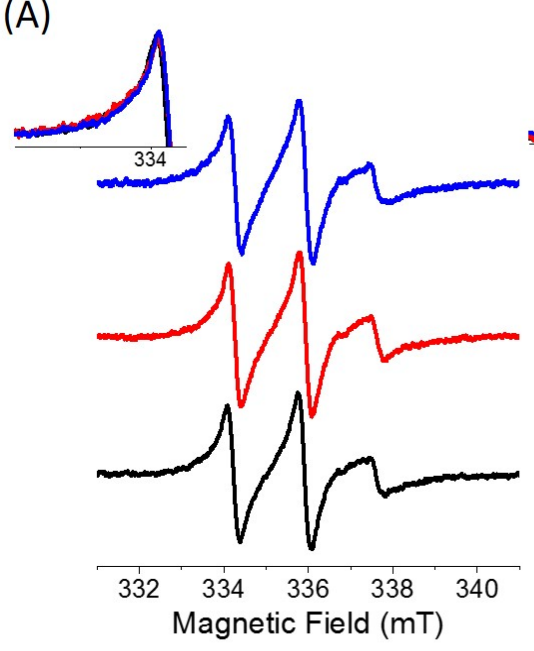

(B)

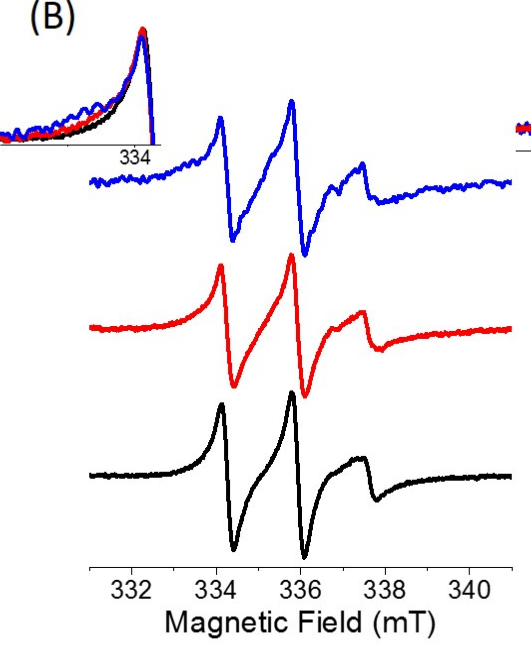

(C)

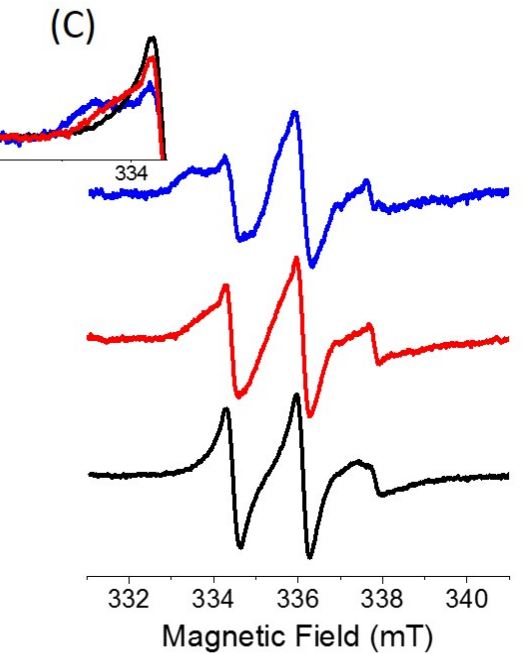

Fig. 3. EPR spectra of SL-PS before (black) and after addition of (A) PEG-(Trp) $)_{4}$, (B) (Trp) 2 -PEG$(\text { DOPA })_{2}$ and (C) PEG-(DOPA) ${ }_{4}$ with $22 \mathrm{mg} / \mathrm{mL}$ (red) and $90 \mathrm{mg} / \mathrm{mL}$ (blue) concentrations. Insets show the low field spectral region to emphasize the formation of signals of covered spin labels.

The second type of spectrum increases with amount of PEG-(DOPA) 4 . Simulations showed that the covered spin labels percentages are about $60 \%$ and $80 \%$ upon addition of $22 \mathrm{mg} / \mathrm{mL}$ and $90 \mathrm{mg} / \mathrm{mL}$ PEG-(DOPA) $)_{4}$. Comparison of EPR spectra of SL-PS after addition of PEG-(DOPA) 4 and (Trp) $)_{2}$-PEG-(DOPA) $)_{2}$ showed that higher DOPA content increases the adhesion. For example, additions of $90 \mathrm{mg} / \mathrm{mL} \mathrm{PEG-(DOPA})_{4}$ and (Trp) $)_{2}$-PEG-(DOPA) $)_{2}$ to SL-PS suspension covered $80 \%$ and $60 \%$ of spin labels on polystyrene nanoparticles, respectively. These results showed that DOPA is crucial for the adhesion. On the other hand, Trp does not bind to PS. However, both Trp and DOPA functionalized PEG polymer show adhesion but weaker than the adhesion of PEG-(DOPA) 4 .

Interactions between DOPA and styrene groups are considered as hydrophobic interactions. In the force free conditions, these hydrophobic interactions are strong enough to adhere DOPA to polystyrene surface. Even though, tryptophan molecules have hydrophobic side chains, their spontaneous interactions with styrene groups are not strong enough for the adhesion. Therefore, PEG-(Trp) ${ }_{4}$ does not adhere to PS surface.

\section{Conclusion}

DOPA molecules show the spontaneous wet adhesive ability. In solution, DOPA modified PEG polymers are able to adhere to the surface of polystyrene nanobeads without applying any force. The hydrophobic interaction between DOPA and the styrene groups on the PS is strong enough to achieve the adhesion. However, another hydrophobic amino acid of Trp cannot accomplish adhesion to PS. The combination of DOPA and Trp functionalized PEG polymer is also able to adhere to PS but less than the adhesion of PEG-(DOPA) 4 to PS. This shows the importance of DOPA amino acids for the wet spontaneous adhesion. EPR spectroscopy allows us to study the spontaneous adhesion of polymers in solution. Increasing the rotational correlation time of the spin centers on the model surface reports the adhesion of DOPA. 


\section{Acknowledgements}

This work was financially supported by Turkish Scientific and Technological Research Council (TUBITAK) via 3501 Program under grant $114 \mathrm{Z} 318$.

\section{References}

[1] B. P. Lee, P. B. Messersmith, J. N. Israelachvili and J. H. Waite: Annu. Rev. Mater. Res. Vol. 41 (2011), p. 99

[2] M. Krogsgaard, V. Nue and H. Birkedal: Chem. Eur. J. Vol. 22 (2016), p. 844

[3] Q. Lu, E. Danner, J. H. Waite, J. N. Israelachvili, H. Zeng and D. S. Hwang: J. R. Soc. Interface Vol. 10 (2013), p. 20120759

[4] H. Xu, J. Nishida, W. Ma, H. Wu, M. Kobayashi, H. Otsuka and A. Takahara: ACS Macro Lett. Vol. 1 (2012), p. 457

[5] İ. Kırpat, Y. Göksel, E. Karakuş, M. Emrullahoğlu and Y. Akdogan: Mater. Lett. Vol. 205 (2017), p. 48

[6] Y. Akdogan, W. Wei, K. Y. Huang, Y. Kageyama, E. W. Danner, D. R. Miller, N. R. M. Rodriguez, J. W. Haite and S. Han: Angew. Chem. Int. Ed. Vol. 53 (2014), p. 11253

[7] K. Dyrek and M. Che: Chem. Rev. Vol. 97 (1997), p. 305

[8] G. Jeschke: BBA-Bioenergetics Vol. 1707 (2005), p. 91

[9] Y. Akdogan, Y. Wu, K. Eisele, M. Schaz, T. Weil and D. Hinderberger: Soft Matter Vol. 8 (2012), p. 11106

[10] D. Tatlidil, M. Ucuncu and Y. Akdogan: Phys. Chem. Chem. Phys. Vol. 17 (2015), p. 22678

[11] Y. Akdogan, M. Emrullahoğlu, D. Tatlıdil and G. Cakan-Akdogan: Phys. Chem. Chem. Phys. Vol. 18 (2016), p. 22531

[12] Y. Akdogan, V. Anbazhagan, D. Hinderberger and D. Schneider: Biochemistry Vol. 51 (2012), p. 7149

[13] E. Erdem: Nanoscale Vol. 9 (2017) p. 10983

[14] S. Stoll, A. Schweiger: J. Magn. Reson. Vol. 178 (2006), p. 42 